\title{
Electrical conductivity relaxation and nuclear magnetic resonance of $\mathrm{Li}$ conducting $\mathrm{Li}_{0.5} \mathrm{La}_{0.5} \mathrm{TiO}_{3}$
}

\author{
C. León, M. L. Lucía, and J. Santamaría \\ Departamento de Física Aplicada III, Facultad de Ciencias Físicas, Universidad Complutense de Madrid, avenida Complutense, \\ E-28040 Madrid, Spain \\ M. A. París and J. Sanz \\ Instituto de Ciencia de Materiales del Consejo Superior de Investigaciones Científicas, Cantoblanco, 28049 Madrid, Spain
}

\author{
A. Várez \\ Departamento de Ingeniería, Escuela Politécnica Superior, Universidad Carlos III de Madrid, 28911 Leganés, Spain
}

(Received 15 August 1995; revised manuscript received 21 February 1996)

\begin{abstract}
Lithium ionic conductivity of $\mathrm{Li}_{0.5} \mathrm{La}_{0.5} \mathrm{TiO}_{3}$ has been studied using nuclear magnetic resonance (NMR) and admittance spectroscopy (AS) techniques. Spin-lattice relaxation and electrical conductivity relaxation are well described in terms of stretched-exponential correlation functions in the time domain of the form $\phi(t)=\exp \left(-(t / \tau)^{\beta}\right)$, but showing different relaxation times scales $\left(\tau_{0}=1.4 \times 10^{-11} \mathrm{~s}\right.$ from NMR and $\tau_{0}=10^{-14} \mathrm{~s}$ from AS), and activation energies ( 0.15 and $0.4 \mathrm{eV}$, respectively). Different $\beta$ exponents, 1 from spin lattice relaxation and 0.4 from electric-field relaxation have been also deduced. A microscopic activation energy for lithium motion of $0.15 \mathrm{eV}$ is deduced from both techniques. Discrepancies between both techniques are analyzed and discussed in terms of frequency-dependent correlation effects. [S0163-1829(96)00225-1]
\end{abstract}

\section{INTRODUCTION}

Interest in $\mathrm{Li}^{+}$ionic conductors has increased in recent years due to their potential applications in solid-state batteries. Recently, high values of the ionic conductivity $\left[10^{-3}(\Omega \mathrm{cm})^{-1}\right.$ at room temperature $]$ have been reported for $\mathrm{Li}$ containing oxides $\mathrm{Li}_{0.5-3 x} R_{0.5+x} \mathrm{TiO}_{3}$ with perovskite-related structure. ${ }^{1-3}$ The maximum conductivity occurs for $R=\mathrm{La}$ and for $x=0.07$, its high values being due to $\mathrm{Li}$ ions partially occupying equivalent $A$ sites of the $A B \mathrm{O}_{3}$ perovskite structure. The presence of many vacant $A$ sites facilitates $\mathrm{Li}$ ions to move easily through them.

Structure data and dc conductivity measurements have already been published, ${ }^{2}$ however, to the best of our knowledge, nuclear magnetic resonance (NMR) measurements and a study of the frequency dependence of the conductivity to explore correlation effects among charge carriers have not been yet performed in these compounds. A combined NMR and electrical conductivity relaxation (ECR) study can be helpful in obtaining information about the dynamics of the transport process. Recently, $\mathrm{Ngai}^{4}$ has presented a comparison between NMR and ECR measurements on Li glassy ionic conductors showing that, although stretchedexponential decays, $\phi(t)=\exp \left(-(t / \tau)^{\beta}\right)$, account for the correlation functions of the relaxation process in both techniques, the exponents $(\beta)$ and the activation energies $(E)$ for the correlation time $(\tau)$ are different in each case. The relationship $\beta_{s} E_{s}=\beta_{\sigma} E_{\sigma}=E_{a}$, where subscripts $s$ and $\sigma$ refer to NMR and ECR, respectively, experimentally established for glassy ionic conductors, has permitted obtaining a singleion microscopic activation energy $\left(E_{a}\right)$ in which both techniques agree. In this paper we have investigated this relationship in samples with a $0.5 \mathrm{Li}$ content per formula $\left(\mathrm{Li}_{0.5} \mathrm{La}_{0.5} \mathrm{TiO}_{3}\right)$. This constitutes a very attractive scenario since we are dealing with crystalline $\mathrm{Li}$ ion conductors and, unlike glasses, not much attention has been paid to the study of correlation effects in crystalline systems.

\section{EXPERIMENTAL}

Samples were prepared heating a stoichiometric mixture of high purity $\mathrm{Li}_{2} \mathrm{CO}_{3}, \mathrm{La}_{2} \mathrm{O}_{3}$ and $\mathrm{TiO}_{2}$ reagents at $120^{\circ} \mathrm{C}$. The reacted power was pelleted and fired at $1350{ }^{\circ} \mathrm{C}$ in air for several hours $(5-11 \mathrm{~h})$ and then quenched to room temperature. The metal molar ratio was determined by inductively coupled-plasma spectroscopy using a JY-70 PLUS spectrometer. Structure was verified by $\mathrm{x}$-ray and electron diffraction.

The ${ }^{7} \mathrm{Li}$ NMR spectra were recorded with an SXP 4/100 Bruker spectrometer. The frequency used for lithium was $\nu_{0}=31.01 \mathrm{MHz}$ that corresponds to an external magnetic field of $B_{0}=1.87 \mathrm{~T}$. The number of accumulations was selected between 100 and 200. In all cases, the signal-to-noise ratio was higher than 10 . A $\pi / 2$ pulse of $2.4 \mu \mathrm{s}$ and a period between successive accumulations higher than five times the spin-lattice relaxation time $\left(T_{1}\right)$ was used for each temperature. Experiments were made between 100 and $530 \mathrm{~K}$. Determination of the $T_{1}$ values was carried out by using a classical $\pi-\tau-\pi / 2$ sequence. ${ }^{5}$ When the intrinsic linewidth was higher than the inhomogeneity of the magnetic field, the spin-spin relaxation times $\left(T_{2}\right)$ were deduced from a single pulse experiment: ${ }^{5}$ if the line shape was Gaussian the $1 / T_{2}$ value was calculated as $0.6 \pi$ times the full width at half height (FWHH), if the line shape was Lorentzian the $1 / T_{2}$ value was calculated as $\pi$ times FWHH. In intermediate cases, an average of the aforementioned values was used. When the intrinsic linewidth was of the same order or smaller than the field inhomogeneity, the determination of $1 / T_{2}$ values was performed by using the Meiboom-Gill modification the Carr-Purcell sequence: ${ }^{6}(\pi / 2)_{x^{\prime}}-\tau$ 


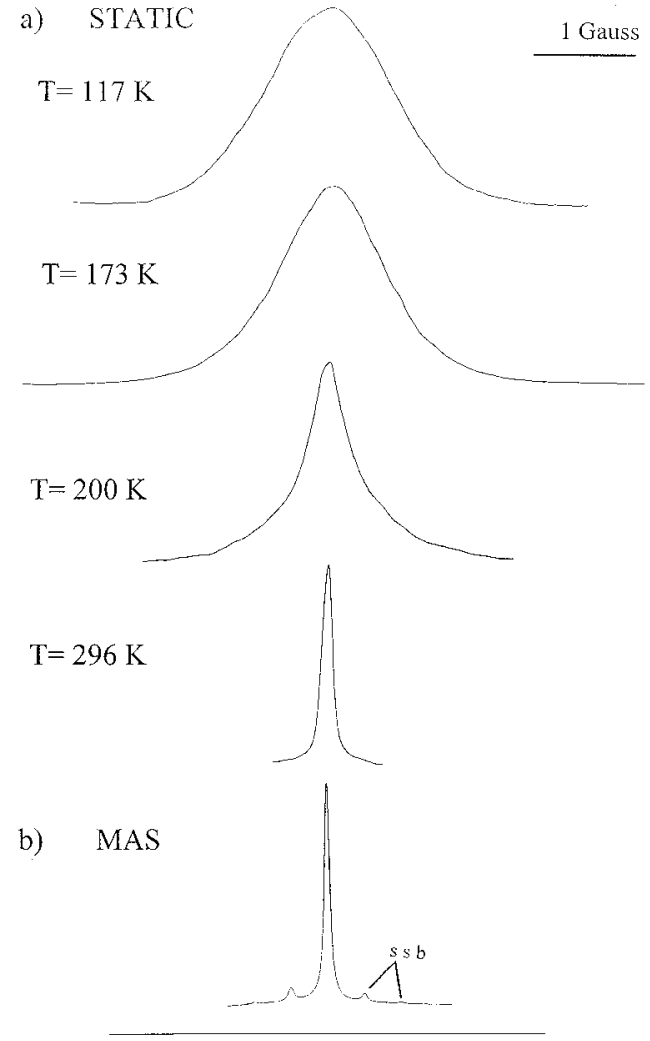

Ho

FIG. 1. (a) Temperature dependence of the static ${ }^{7} \mathrm{Li}$ NMR spectra recorded at $31.01 \mathrm{MHz}$. (b) ${ }^{7} \mathrm{Li}$ MAS spectrum recorded at a spinning rate of $500 \mathrm{~Hz}$ at room temperature. ssb denote spinning side bands.

$-\left[(\pi)_{y^{\prime}}-\tau-(\pi)_{y^{\prime}}\right]^{n} . \quad{ }^{7} \mathrm{Li}$ magic-angle-spinning (MAS) spectra were recorded using a MAS Bruker MSL/400 spectrometer at a spinning rate of $500 \mathrm{~Hz}$.

Admittance spectroscopy was measured in the frequency range $20 \mathrm{~Hz}-1 \mathrm{MHz}$ using an automatically controlled $\mathrm{HP}$ 4284A precision LCR meter, at temperatures comprised between 150 and $330 \mathrm{~K}$. The samples were cylindrical pellets 5 $\mathrm{mm}$ in diameter and $0.7 \mathrm{~mm}$ thick on whose faces gold electrodes were deposited by evaporation. Measurements were conducted under an $\mathrm{N}_{2}$ flow to ensure an inert atmosphere.

\section{RESULTS AND DISCUSSION}

\section{A. Nuclear magnetic resonance (NMR)}

The static ${ }^{7} \mathrm{Li}$ NMR spectra $(I=3 / 2)$, recorded at increasing temperatures [Fig. 1(a)], show only one line centered at the $\mathrm{Li}$ resonance frequency, that progressively narrows as temperature increases not showing the $(1 / 2,3 / 2)$ transitions due to quadrupolar interaction of $\mathrm{Li}$ with its environment. ${ }^{7}$ In order to exclude the presence of masked satellite peaks associated with these transitions the spectrum was recorded under magic-angle-spinning (MAS) conditions at room temperature [see Fig. 1(b)]. The absence of outer static transitions was thus confirmed concluding that $\mathrm{Li}$ occupies sites with small static quadrupole interactions, i.e., high symmetry sites.

At temperatures below $200 \mathrm{~K}$ the line shape is Gaussian and the linewidth is almost constant, indicating that interac- tions of $\mathrm{Li}$ with surrounding atoms are constant and $\mathrm{Li}$ remains at times $\tau>\left(\left\langle\Delta \omega^{2}\right\rangle\right)^{1 / 2}$ in fixed positions. $\left\langle\Delta \omega^{2}\right\rangle$ is the second moment of the spectrum recorded at low temperatures and can be calculated from the spin-spin relaxation time, with the expression: ${ }^{5}\left\langle\Delta \omega^{2}\right\rangle=2 /\left(T_{2}\right)^{2}$. From the $1 / T_{2}$ measured at low temperatures a value of $\left\langle\Delta \omega^{2}\right\rangle$ $=4.25 \times 10^{7} \mathrm{~s}^{-2}$ has been deduced.

Since static quadrupolar interactions are not present, the linewidth of the spectra must be mainly due to dipole interactions. In particular, contributions of $\mathrm{Li}-\mathrm{Li}$ interactions should be preponderant since atoms surrounding $\mathrm{Li}$ ions, other than Li itself, exhibit small magnetic moments. On this basis, the second moment of the powder sample could be calculated as ${ }^{7}$

$$
\left\langle\Delta \omega_{I-I}^{2}\right\rangle=\frac{3}{5} \gamma_{I}^{4} \hbar^{2} I(I+1) \sum \frac{1}{r_{k}^{6}},
$$

where $\hbar$ is the Planck's constant, $\gamma_{I}$ is the giromagnetic constant for ${ }^{7} \mathrm{Li}, I=3 / 2$ its nuclear spin, and $r_{k}$ is the distance between the interacting spins.

If only nearest- and next-nearest $\mathrm{Li}$ neighbors are assumed to contribute to line broadening [additional contributions represent only $5 \%$ of $\left\langle\Delta \omega^{2}\right\rangle$ value], and assuming that lithium distributes randomly over the $A$ positions of the perovskite structure, the former expression can be written as

$$
\left\langle\Delta \omega_{I-I}^{2}\right\rangle=2.92 \times 10^{10}\left[P \cdot Z \frac{1}{a^{6}}+P \cdot Z^{\prime} \frac{1}{(\sqrt{2} a)^{6}}\right] \mathrm{s}^{-2},
$$

where $Z=6$ and $Z^{\prime}=12$ are the number of first- and secondnearest $A$ positions surrounding an $A$ site. $P=1 / 2$ is the probability of occupation of sites $A$ by $\mathrm{Li}$ and $a$ is the distance in $\AA$ between adjacent $A$ sites. Introducing the experimental second moment in Eq. (2), a value of $a=3.7 \AA$ is obtained, which is very close to the structural distance $\left(a_{p}=3.8 \AA\right)$ calculated from x-ray-diffraction experiments.

At temperatures higher than $200 \mathrm{~K}$, the line shape becomes Lorentzian and the linewidth decreases as a consequence of $\mathrm{Li}$ motion which induces a progressive cancellation of interactions of $\mathrm{Li}$ with its environment. In this case, the local fields are changing with time and only an averaged value is observed by nuclei. This phenomenon happens when the correlation time for $\mathrm{Li}$ motion $\tau$ is smaller than $\left(\left\langle\Delta \omega^{2}\right\rangle\right)^{1 / 2}$ (motional narrowing effect). From the variation of $1 / T_{2}$ vs $1000 / T$, plotted in Fig. 2, an activation energy for Li motion $E_{s}=0.15 \mathrm{eV}$ has been calculated.

On the other hand, an activation energy of Li motion can also be obtained from analysis of $1 / T_{1}$ data. A plot of $1 / T_{1}$ values vs $1000 / T$ is presented in Fig. 2. At temperatures below $145 \mathrm{~K}, 1 / T_{1}$ values decrease slowly towards a plateau not shown in the figure. Above this temperature, $1 / T_{1}$ increases attaining a maximum at $320 \mathrm{~K}$. Activation energies calculated at both sides of $1 / T_{1}$ plot yielded the same value, $E_{s}=0.15 \mathrm{eV}$, which also coincides with that deduced from $1 / T_{2}$ measurements. The solid line in Fig. 2 is the fit of $1 / T_{1}$ data to the symmetrical curve given by the Bloembergen-Purcell-Pound (BPP) expression ${ }^{8}$ 


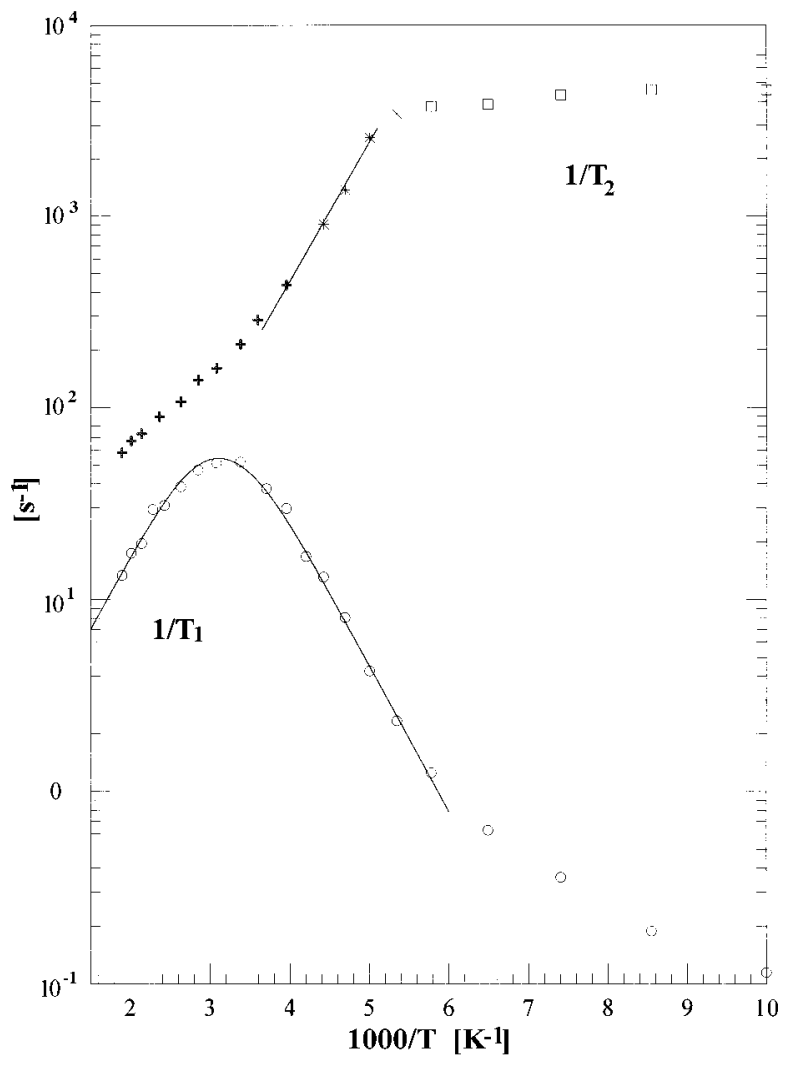

FIG. 2. Experimental temperature dependence of the spin-lattice relaxation rates $1 / T_{1}(\bigcirc)$, and spin-spin relaxation rates $1 / T_{2}$. The method used for determination of $1 / T_{2}$ are (1) one pulse: ( $\square$ ) Gaussian line shape, $(X)$ intermediate Gaussian-Lorentzian line shape, (*) Lorentzian line shape; (2) CP-MG sequence: (+) Lorentzian line shape.

$$
\frac{1}{T_{1}}=C\left(\frac{\tau}{1+\left(\omega_{0} \tau\right)^{2}}+4 \frac{\tau}{1+4\left(\omega_{0} \tau\right)^{2}}\right),
$$

where $\tau=\tau_{0 s} \exp \left(E_{s} / k T\right)$ is the correlation time, $E_{s}$ is the activation energy for $\mathrm{Li}$ motion, and $C$ depends on the mechanism responsible for the spin-lattice relaxation (SLR) rate. Note that Eq. (3) is the result of the Li motion described by the correlation function $\phi(t)=\exp \left(-(t / \tau)^{\beta}\right)$, with $\beta=1$. The values of $\tau_{0 s}$ and $E_{s}$ best reproducing experimental results are presented in Table I.

It is generally admitted that there are three major NMR mechanisms which cause SLR in ionic solids: (1) dipole relaxation, generated by fluctuating interactions between nuclear magnetic moments of atoms; (2) quadrupolar relaxation, due to the coupling between the nuclear quadrupole moment and fluctuating electric-field gradients (EFG) created at nuclear sites; and (3) paramagnetic relaxation, caused by coupling of the magnetic moments of nuclei and paramagnetic centers. If the SLR rate were dominated by the dipole interaction between lithium ions in $\mathrm{Li}_{0.5} \mathrm{La}_{0.5} \mathrm{TiO}_{3}$, the $C$ value obtained from the fitting of the $1 / T_{1}$ experimental data $\left(C=7.4 \times 10^{9} \mathrm{~s}^{-2}\right)$ should be equal to $C$ $=(2 / 3)\left\langle\Delta \omega_{I-I}^{2}\right\rangle,{ }^{7}$ where $\left\langle\Delta \omega_{I-I}^{2}\right\rangle$ is the static second moment obtained from the spectra recorded at low temperatures. However, this expression yields a $C$ value $\left(C=2.8 \times 10^{7}\right.$ $\mathrm{s}^{-2}$ ) which is two orders of magnitude lower than the one
TABLE I. Results of the fitting of SLR and ECR to a stretchedexponential decay in the time domain of the form $\exp \left(-(t / \tau)^{\beta}\right)$. $\tau$ is thermally activated with an activation energy $E$ and a preexponential factor $\tau_{0}$. A microscopic activation energy $E_{a}$ is deduced as the product $\beta E$.

\begin{tabular}{lcrlr}
\hline \hline & $\tau_{0}(\mathrm{~s})$ & $E(\mathrm{eV})$ & $\beta$ & $E_{a}(\mathrm{eV})$ \\
\hline NMR & $1.4 \pm 0.1 \times 10^{-11}$ & $0.15 \pm 0.01$ & 1 & $0.15 \pm 0.01$ \\
ECR & $1.0 \pm 0.3 \times 10^{-14}$ & $0.4 \pm 0.02$ & 0.4 & $0.16 \pm 0.01$ \\
\hline \hline
\end{tabular}

deduced from experimental data, indicating that other mechanisms must be responsible for the SLR rate. As quoted previously, the analysis of spectra does not reveal the existence of static quadrupole interactions capable of producing quadrupole splitting. However, fluctuating EFG associated with $\mathrm{Li}$ motion could induce quadrupole relaxation ${ }^{7}$ of $\mathrm{Li}$ nuclei. In this case, $C \approx\left(2 \pi^{2} / 5\right) C_{Q}^{2}$, and the quadrupole coupling constant $\left(C_{Q}\right)$ causing SLR should be $\approx 43 \mathrm{kHz}$, but the contribution to line shape of these fluctuating interactions should be averaged out by Li motions. Finally, another source that might explain the relaxation behavior is the existence of small amounts of paramagnetic impurities introduced by oxygen stoichiometry deviations observed by other authors in the same type of compounds. ${ }^{1}$ In particular, assuming the existence of rapid spin-energy diffusion for the SLR rate, ${ }^{9}$ the presence of $0.4 \%$ of paramagnetic centers would explain the observed $C$ value. However, its contribution to the second moment of the spectrum would be only $2 \%$ from that experimentally determined at low temperatures and, therefore, very difficult to be detected from the spectrum analysis. Whatever the SLR is caused by paramagnetic impurities or by quadrupole interaction associated with charge fluctuations caused by Li motion, expression (3) would be valid and the same correlation times and activation energies would be deduced in both cases. On this basis, the temperature at which the maximum of $1 / T_{1}$ appears would be directly related to Li motion and not to the type of interaction causing the relaxation.

\section{B. Admittance spectroscopy}

The real part of the conductivity $(\sigma)$, related to the imaginary part of the permittivity $\left(\varepsilon^{\prime \prime}\right)$ through $\sigma=\varepsilon^{\prime \prime} \omega$, is presented in Fig. 3 as a function of frequency for different temperatures. The rapid decrease of the conductivity at low frequency and high temperatures is caused by blocking of charge carriers at grain boundaries and electrodes, and shifts to lower frequencies when the temperature is decreased. Since the grain-boundary conductivity is slightly stronger activated than the bulk one, one can obtain information about the bulk free from the effect of blocking mechanisms in the available frequency window. The whole analysis presented in this paper has been conducted at temperatures lower than $235 \mathrm{~K}$ to avoid the above-mentioned blocking effects.

The plateau observed in conductivity plots can thus be ascribed to the bulk conductivity and a crossover occurs to a power-dependent regime. This dispersive behavior can be expressed as

$$
\sigma^{*}(\omega)=\sigma_{\mathrm{dc}}\left[1+\left(j \omega / \omega_{p}\right)^{n}\right]+j \omega \varepsilon_{\infty},
$$




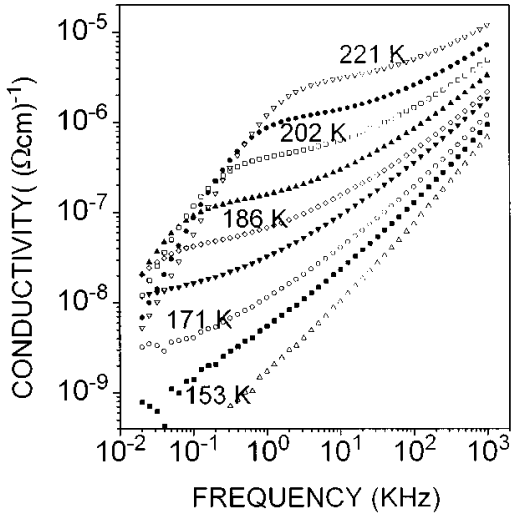

FIG. 3. Real part of the conductivity vs frequency at several temperatures $(221,211,202,193,186,179,171,164$, and $153 \mathrm{~K})$. The conductivity shows a power-law behavior at high frequencies, according to $\sigma^{*}(\omega)=\sigma_{\mathrm{dc}}\left[1+\left(j \omega / \omega_{p}\right)^{n}\right]+j \omega \varepsilon_{\infty}$.

where $\sigma_{\mathrm{dc}}$ is the bulk conductivity, $\omega_{p}$ is the crossover frequency, both being thermally activated with the same activation energy, and $\varepsilon_{\infty}$ is the high-frequency permittivity. The fractional exponent $n$ is related to the degree of correlation among moving ions ( $n$ equals zero for a completely uncorrelated motion). This behavior has been widely observed in structurally disordered solid electrolytes and also in crystalline materials, and constitutes the main feature of the socalled "universal dielectric response," 10 which appear as a result of a nonrandom hopping conduction mechanism.

The power-law dependence of the conductivity in the frequency domain seems to be linked to stretched-exponential decay functions in the time domain. It has been found empirically for many glassy ionic conductors, that the decrease of the electric field at a constant displacement vector can be well described by the Kohlrausch-Williams-Watts (KWW) function $^{11}$

$$
\phi(t)=\exp \left(-\left(t / \tau_{\sigma}\right)^{\beta}\right),
$$

where $\tau_{\sigma}$ is thermally activated with the same activation energy as the dc conductivity, $\beta$ is associated with correlated ion hopping.

Time response is customarily obtained from the dielectric modulus $M^{*}(\omega)=1 / \varepsilon^{*}(\omega)=j \omega / \sigma^{*}(\omega)$, which can be expressed in terms of the time derivative of the decay function according to expression (6):

$$
M^{*}(\omega)=\frac{1}{\varepsilon_{\infty}}\left[1-\int_{0}^{\infty}\left(-\frac{d \phi}{d t}\right) e^{-j \omega t} d t\right] .
$$

The modulus formalism is widely used in the analysis of complex admittance data because of its relative insensitivity to blocking phenomena and because it offers a convenient way to compare electrical conductivity relaxation (ECR) with spin-lattice relaxation (SLR) data.

Figure 4 shows the imaginary part of the modulus for various temperatures; solid lines are fits to expression (6) following the method proposed by other authors. ${ }^{12,13}$ The high-frequency permittivity $\varepsilon_{\infty}$ obtained from the fits had a practically temperature-independent value of $(7.5 \pm 0.5) 10^{-12} \mathrm{~F} / \mathrm{cm}$, in good agreement with the one de-

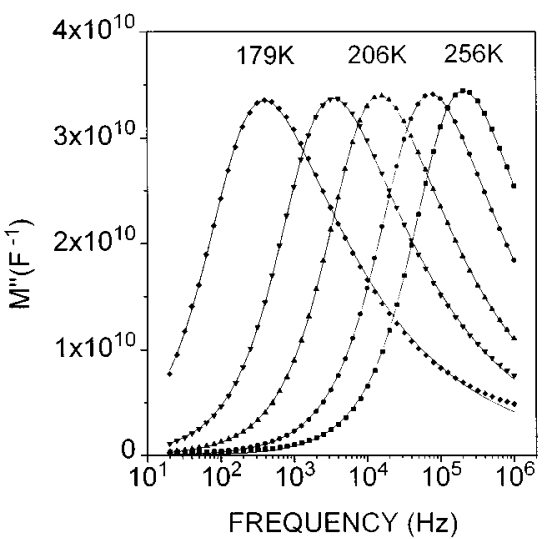

FIG. 4. Imaginary part of the dielectric modulus vs frequency in a semi-log scale at 179, 193, 206, 221, and $235 \mathrm{~K}$. Solid lines are fits to expression (5), showing KWW decays in the time domain.

termined from the real capacitance at high frequencies and low temperatures. In the temperature range (179-235 K) where the modulus peak was observed, $\beta$ values close to 0.4 were obtained, showing a slight temperature dependence. $\beta$ and $1-n$ values resulting from the fits to modulus and conductivity plots, respectively, are plotted together in Fig. 5 as a function of temperature. $\beta$ values agree with $1-n$ suggesting that $n$ and $\beta$ may be reflecting the same physical situation: they are different descriptions of the effect of correlation between moving species. A decrease is observed in $1-n$ for temperatures lower than $180 \mathrm{~K}$; no data points of $\beta$ are presented for this temperature range since its determination requires a well-resolved modulus peak in the available frequency window and below $180 \mathrm{~K}$ the peak is shifted to frequencies lower than $20 \mathrm{~Hz}$, the low-frequency limit of the impedance analyzer used in this work. This decrease has been observed previously, ${ }^{14}$ and it may be explained in terms of a transition to a strongly correlated regime in which ions behave more in a dipolelike fashion ( $n$ tends to 1 ) than as free carriers. In fact the spin-lattice relaxation rate (see Fig. 2) shows that the Li mobility is activated for temperatures higher than $180 \mathrm{~K}$, the onset of the decrease in $1-n$. The study of this effect is out of the scope of this work and we

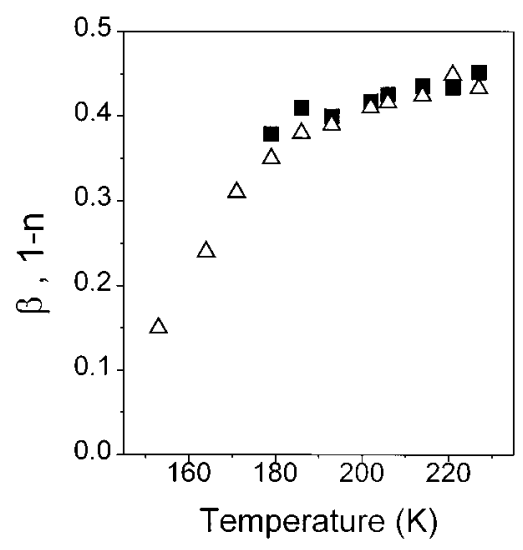

FIG. 5. Temperature dependence of $\beta(\mathbf{\square})$ and $1-n(\triangle)$ showing the equivalence of time and frequency domain pictures. 


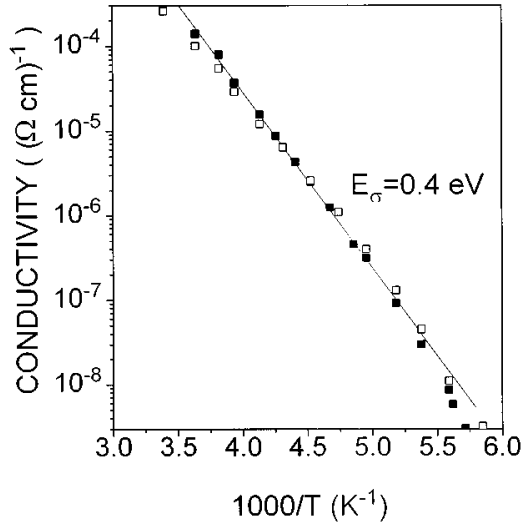

FIG. 6. Dc conductivity vs $1000 / T$ as obtained from the modulus formalism (solid symbols) and as deduced from conductivity vs frequency plot (open symbols).

have focused the discussion in the better known $1-n$ close to the 0.4 regime.

Dc conductivity can be obtained fitting expression (4) to conductivity data or expression (6) to modulus data. From the fits of modulus data to expression (6) the dc conductivity can be calculated as $\sigma_{0}=\varepsilon_{\infty} /\left\langle\tau_{\sigma}\right\rangle$ where $\varepsilon_{\infty}$ is the highfrequency dielectric permittivity $\left(7.5 \times 10^{-12} \mathrm{~F} / \mathrm{cm}\right)$ and $\left\langle\tau_{\sigma}\right\rangle$ is the mean relaxation time obtained as $\left\langle\tau_{\sigma}\right\rangle=\int_{0}^{\infty} \phi(t) d t=\tau_{\sigma} \Gamma(1 / \beta) / \beta$, where $\Gamma$ is the Euler's gamma function. ${ }^{13}$ An Arrhenius plot of the so-obtained dc conductivity data is presented in Fig. 6. The same figure displays de conductivity values obtained directly from conductivity vs frequency plot, in good agreement with those obtained from the modulus formalism. An activation energy of $0.4 \mathrm{eV}$ is deduced for the dc conductivity.

\section{Comparison of SLR and ECR data}

The temperature dependence of the dielectric modulus and the impedance offers a direct way to compare electrical conductivity relaxation with spin-lattice relaxation data. The modulus is related to the Fourier transform of the time derivative of the decay function [denoted $\hat{\Phi}(\omega)$ hereafter] as quoted by expression (6). Since $\hat{\Phi}(\omega)=j \omega \hat{\Phi}(\omega)-1$ and $M^{*}(\omega)=j \omega Z^{*}(\omega)$ the impedance $Z^{*}(\omega)$ can be directly written in terms of the Fourier transform of the decay function: $\hat{\Phi}(\omega)=\varepsilon_{\infty} Z^{*}(\omega)$.

On the other hand, the spin-lattice relaxation time $1 / T_{1}$ is connected with the spectral density function $J(\omega, T)$ through the following expression:

$$
\frac{1}{T_{1}(\omega, T)}=A\left[J^{\prime}(\omega, T)+4 J^{\prime}(2 \omega, T)\right] .
$$

The general trends of $1 / T_{1}$ are much the same than those of $J^{\prime}(\omega, T)$ and

$$
J(\omega, T)=\int_{0}^{\infty} C(t, T) e^{-j \omega t} d t,
$$

where $C(t, T)$ is the correlation function. Comparing NMR and ECR results usually consists of comparing the spectral
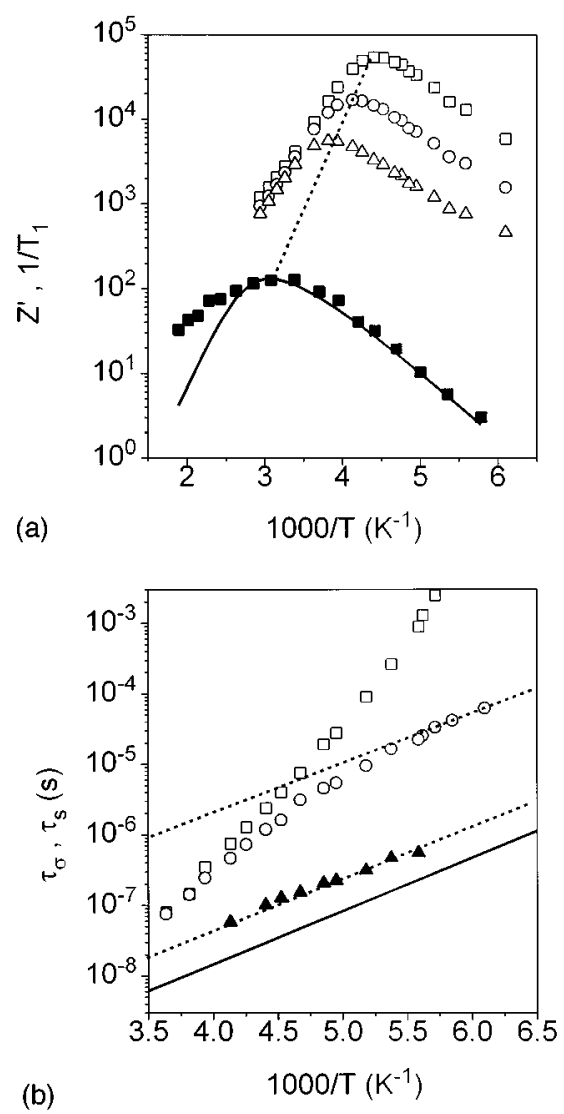

FIG. 7. (a) Temperature dependence of the real part of the specific impedance in $\Omega \mathrm{cm}$ at $(\square) 10^{5} \mathrm{~Hz},(\bigcirc) 3.210^{5} \mathrm{~Hz}$, and $(\triangle) 10^{6} \mathrm{~Hz}$ and $1 / T_{1}(\boldsymbol{\square})$. The solid line is the extrapolation of the impedance data to $31 \mathrm{MHz}$, the NMR measuring frequency. $1 / T 1$ data have been shifted vertically to match impedance data. The dotted line connecting impedance maxima is activated with $0.4 \mathrm{eV}$, the dc activation energy. (b) Temperature dependence of the conductivity relaxation time $\tau_{\sigma}=\varepsilon_{\infty} / \sigma_{\mathrm{dc}}(\square)$ and of the NMR correlation time $\tau_{s}$ (solid line) showing different time scales and activation energies. $(\bigcirc)$ represent an ac conductivity relaxation time $\tau_{\mathrm{ac}}=\varepsilon_{\infty} / \sigma_{\mathrm{ac}}$ calculated at $10^{5} \mathrm{~Hz}$. ( $\left.\boldsymbol{\Delta}\right)$ is an ac relaxation time extrapolated at $31 \mathrm{MHz}$. Note that lines and symbols in (a) and (b) have different meanings.

shape of the decay function $\phi(t)$ and the correlation function $C(t)$. And many examples exist in the literature in which both show stretched-exponential dependences of the form $\exp \left(-(t / \tau)^{\beta}\right)$ but with different $\beta$ values and activation energies for the correlation time deduced from each technique. ${ }^{4,15}$

Figure 7(a) shows the temperature dependence of the real part of the impedance at various frequencies together with the $1 / T_{1}$ curve. Impedance data have been extrapolated to obtain the curve (solid line) corresponding to a frequency of $31 \mathrm{MHz}$ (NMR measuring frequency). The impedance curve so obtained can be compared with the spin-lattice relaxation data. The $1 / T_{1}$ curve has been displaced vertically to bring the maxima to coincide. It can be readily seen that the maxima occur at the same temperature in both cases. At the low-temperature side of the maximum ECR and NMR show practically the same activation, but in the high-temperature side they show a markedly different behavior: while impedance characteristics are strongly asymmetric, in agreement 
with the KWW dependence in the time domain, $1 / T_{1}$ data show a nearly symmetric characteristic. This agrees with the $\beta$ values of 0.4 and 1 obtained from ECR and NMR data, respectively. This result seems to support the idea proposed by several authors ${ }^{16}$ that ECR and SLR are governed by different correlation functions.

Following Ngai's coupling model, ${ }^{17}$ a single-ion microscopic activation energy can be obtained from ECR and NMR data. ${ }^{4}$ This model is based on the assumption that at sufficiently short times the relaxation process occurs independently for each ion. The single-ion relaxation rate is activated with a microscopic activation energy $E_{a}$ which is related to the activation energy of the conductivity $E_{\sigma}$ through $E_{a}=\beta_{\sigma} E_{\sigma}$, and to the activation energy deduced from the spin-lattice relaxation $E_{s}$ through $E_{a}=\beta_{s} E_{s}$. In this way, a microscopic activation energy for Li motion of 0.16 $\mathrm{eV}$ has been obtained from ECR in good agreement with the $0.15 \mathrm{eV}$ obtained from NMR. Interestingly, although both techniques yield practically the same microscopic activation energy, they show a remarkable disagreement in $\beta$ values $\left(\beta_{\sigma}\right.$ and $\left.\beta_{s}\right)$ and in activation energies for long-range motion $\left(E_{\sigma}\right.$ and $E_{s}$ ) (see Table I for details). However, the relation $\beta_{s} E_{s}=\beta_{\sigma} E_{\sigma}$, proposed by Ngai, is confirmed to be valid in our case.

Another interesting fact is that ECR and SLR show also a marked disagreement in the correlation times deduced from each technique, see Fig. 7(b). While NMR yields a correlation time $\left[\tau_{s}=\tau_{0 s} \exp \left(E_{s} / k T\right)\right]$ activated with $0.15 \mathrm{eV}$ and $\tau_{0 s}$ of $1.4 \times 10^{-11} \mathrm{~s}$, the relaxation time deduced from conductivity measurements as $\varepsilon_{\infty} / \sigma\left[\tau_{\sigma}=\tau_{0 \sigma} \exp \left(E_{\sigma} / k T\right)\right]$ is activated with $0.4 \mathrm{eV}$ and shows $\tau_{0 \sigma}=10^{-14} \mathrm{~s}$. This discrepancy has been observed previously in superionic glasses ${ }^{14}$ and might be due to the fact that, while $\tau_{\sigma}$ is obtained in terms of a dc conductivity, $\tau_{s}$ is obtained from the SLR rate at the $31 \mathrm{MHz}$ measuring frequency. In fact, if for the purpose of this discussion we define an ac relaxation time as $\tau_{\mathrm{ac}}=\varepsilon_{\infty} / \sigma_{\mathrm{ac}}, \sigma_{\mathrm{ac}}$ being the ac conductivity at a given frequency, we can see that depending on the frequency there will be a temperature driven crossover from a dc to an ac regime. The activation energy changes from $E_{\sigma}$ at high temperatures to $E_{a}$ at the lower temperatures. See, for example, data of $\tau_{\mathrm{ac}}$ at $10^{5} \mathrm{~Hz}$ in Fig. 7(b). Moreover, $\tau_{\mathrm{ac}}$ values at 31 $\mathrm{MHz}$ obtained by extrapolation using expression (3) are also presented in Fig. 7(b) showing values relatively close to those obtained from SLR, and the same activation energy $\left(E_{a} \cong E_{s}\right)$. The transition in the temperature dependence of the correlation time from the ac regime in low temperature to the dc regime in high temperature when one measures the conductivity in the appropriate time scale may provide a clue to understand the $\mathrm{Li}$ conductivity and to reconcile both techniques. Most likely, the origin of this crossover lies in the power-law frequency dependence of the conductivity caused by correlation among moving ions. Some models ${ }^{18}$ have proposed that the effect of those correlations in the hopping conduction mechanism is that mutual interactions may force ions to backhop to their previous position after a hopping event. The experimental value of the ac conductivity is thus dependent on the time scale in the following sense: forwardbackward hopping sequences within the time window can be considered as "unsuccessful" hops. They do not contribute to the dc conductivity observed at low frequency but they contribute to the ac conductivity at a higher frequency $(f)$ as far as they do not prove unsuccessful within a time interval of duration $1 / f$. Cooperative effects would be responsible for the increase observed in the activation energy for long-range ionic transport: while the short-range motion regime at high frequency is activated with $E_{a}$, the dc motion is activated with $E_{\sigma}=E_{a} /(1-n)$. Although this behavior is accounted for by the empirical expression 4 , an explanation for its ultimate physical origin is not possible within the frame of this work. It turns out that, at present, a direct comparison between the results obtained from both techniques is difficult, and as far as measurements are carried out at different frequencies both techniques should be considered complementary rather than equivalent.
${ }^{1}$ Y. Inaguma, L. Chen, M. Itoh, T. Nakamura, T. Uchida, M. Ikuta, and M. Wakihara, Solid State Commun. 86, 689 (1993).

${ }^{2}$ Y. Inaguma, L. Chen, M. Itoh, and T. Nakamura, Solid State Ionics 70/71, 196 (1994).

${ }^{3}$ M. Itoh, Y. Inaguma, W. Jung, L. Chen, and T. Nakamura, Solid State Ionics 70/71, 203 (1994).

${ }^{4}$ K. L. Ngai, Phys. Rev. B 48, 13481 (1993) (and references therein).

${ }^{5}$ E. Fukushima and S. B. W. Roeder, Experimental Pulse NMR ( $a$ Nuts and Bolts Approach) (Addison-Wesley, Reading, MA, 1981).

${ }^{6}$ C. P. Slichter, in Principles of Magnetic Resonance, 3rd ed., edited by P. Fulde (Springer-Verlag, Berlin, 1991).

${ }^{7}$ A. Abragam, in The Principles of Nuclear Magnetism, edited by W. C. Marshall and D. H. Wilkinson (Oxford University Press, Oxford, 1961).

${ }^{8}$ N. Bloembergen, E. M. Purcell, and R. V. Pound, Phys. Rev. 73, 679 (1948).

${ }^{9}$ S. M. Day, G. B. Grimes, Jr., and W. Weatherford, Phys. Rev.
139, 515 (1965).

${ }^{10}$ A. K. Jonscher, in Dielectric Relaxation in Solids (Chelsea Dielectric, London, 1983).

${ }^{11}$ R. Kohlrausch, Ann. Phys. (Leipzig) 72, 393 (1847).

${ }^{12}$ P. B. Macedo, C. T. Moynihan, and R. Bose, Phys. Chem. Glasses 13, 171 (1972).

${ }^{13}$ K. L. Ngai, R. W. Rendell, and H. Jain, Phys. Rev. B 30, 2133 (1984).

${ }^{14}$ W. K. Lee, J. F. Liu, and A. S. Nowick, Phys. Rev. Lett. 67, 1559 (1991).

${ }^{15}$ M. Tatsumisago, C. A. Angell, and S. W. Martin, J. Chem. Phys. 97, 6968 (1992).

${ }^{16}$ M. Meyer, P. Maass, and A. Bunde, Phys. Rev. Lett. 71, 573 (1993).

${ }^{17}$ K. L. Ngai, Comments Solid State Phys. 9, 127 (1979); 9, 141 (1980).

${ }^{18}$ K. Funke, in Superionic Solids and Solid Electrolytes: Recent Trends, edited by A. L. Laskar and S. Chandra (Academic, New York, 1989); Prog. Solid State Chem. 22, 111 (1993). 